Supporting Information

\title{
Graded Channel Junctionless InGaZnO Thin Film Transistors with Both High Transporting Properties and Good Bias Stress Stability
}

Jie Liu, ${ }^{\dagger}$ Jianlei Guo, ${ }^{\dagger}$ Wenlong Yang, ${ }^{\dagger}$ Cuiru Wang, ${ }^{\dagger}$ Bin Yuan, ${ }^{\ddagger}$ Jia Liu,${ }^{\dagger}$ Zhiheng $W u,{ }^{\dagger}$ Qing Zhang, ${ }^{\dagger}$ Dapu Liu, ${ }^{\dagger}$ Huixin Chen, ${ }^{\dagger}$ Yinyin $Y u,{ }^{\dagger}$ Suilin Liu,,${ }^{\S} *$ Guosheng Shao, ${ }^{\dagger}$ and Zhiqiang $Y a o^{\dagger, *}$

†State Centre for International Cooperation on Designer Low-Carbon and Environmental Materials, School of Materials Science and Engineering, Zhengzhou University, Zhengzhou, 450001, China

OLED Product Development Department, Tianma Microelectronics Co., Ltd., Wuhan, 430074, China

${ }^{\S}$ Analytical and Testing Center, Sichuan University, Chengdu, 610064, China

*Corresponding Author: 1s1@scu.edu.cn (S. Liu); zqyao@zzu.edu.cn (Z. Yao) 


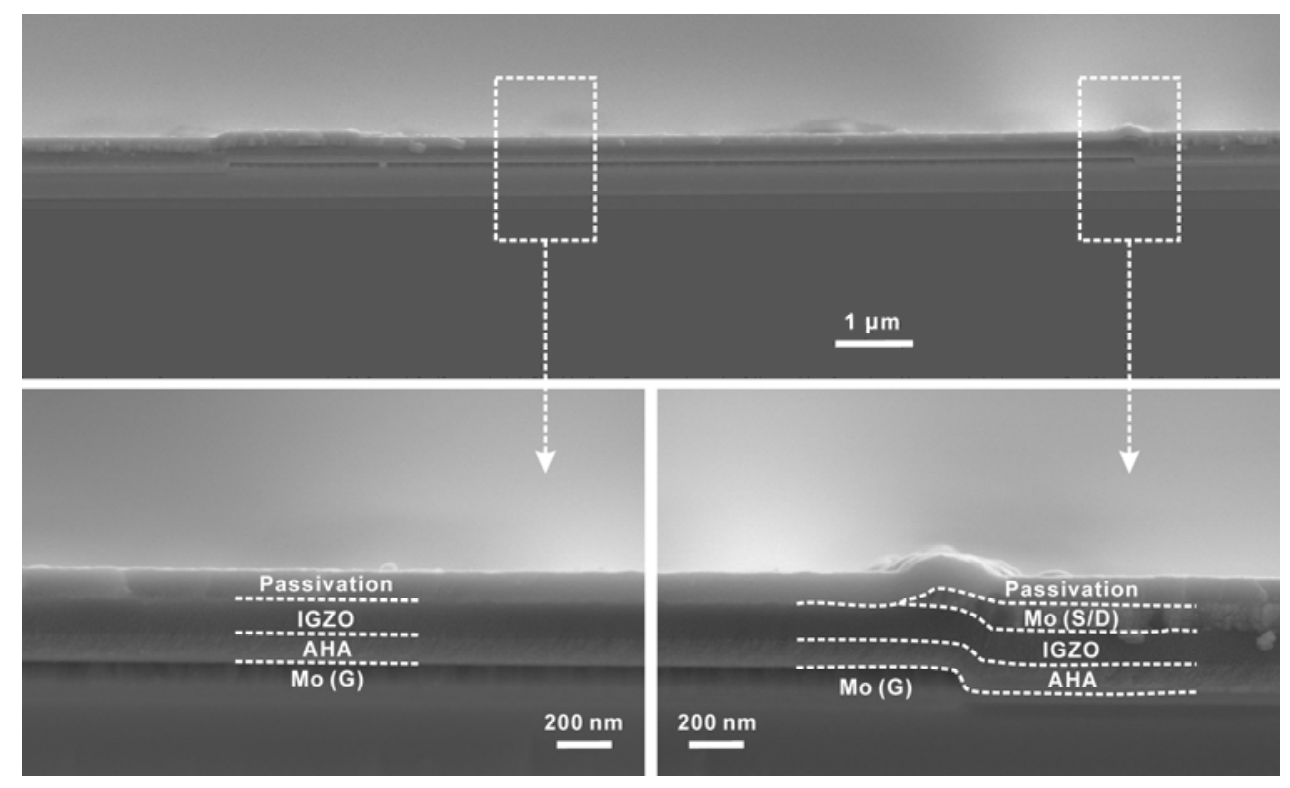

Figure S1. Cross-sectional SEM images of the bottom-gate top-contact IGZO TFTs. 
(a)

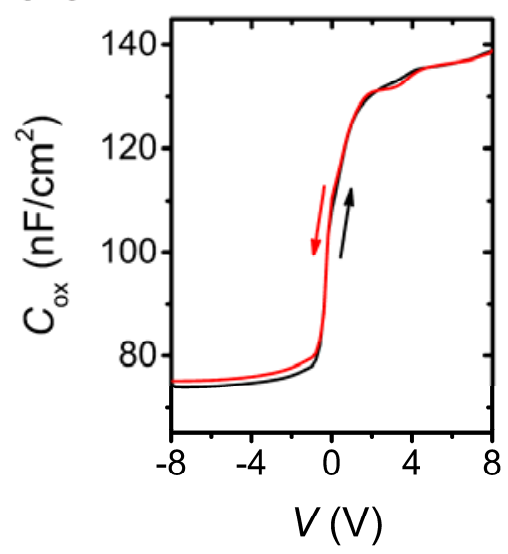

(b)

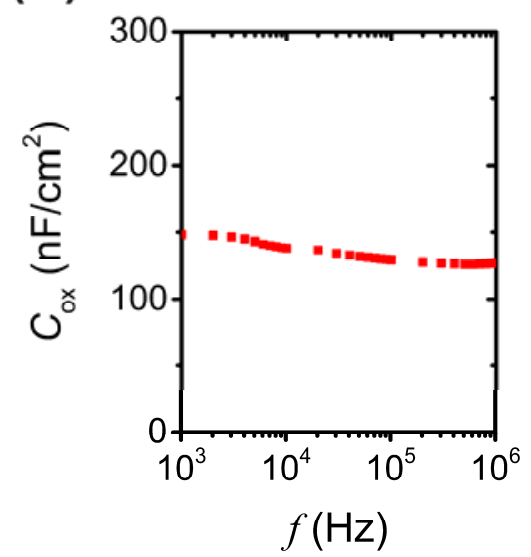

Figure S2. Dielectric performance of the AHA gate insulator: (a) $C-V$ measurements at 10 $\mathrm{kHz}$; (b) $C-f$ measurements with $f$ varying from $1 \mathrm{kHz}$ to $1 \mathrm{MHz}$. 


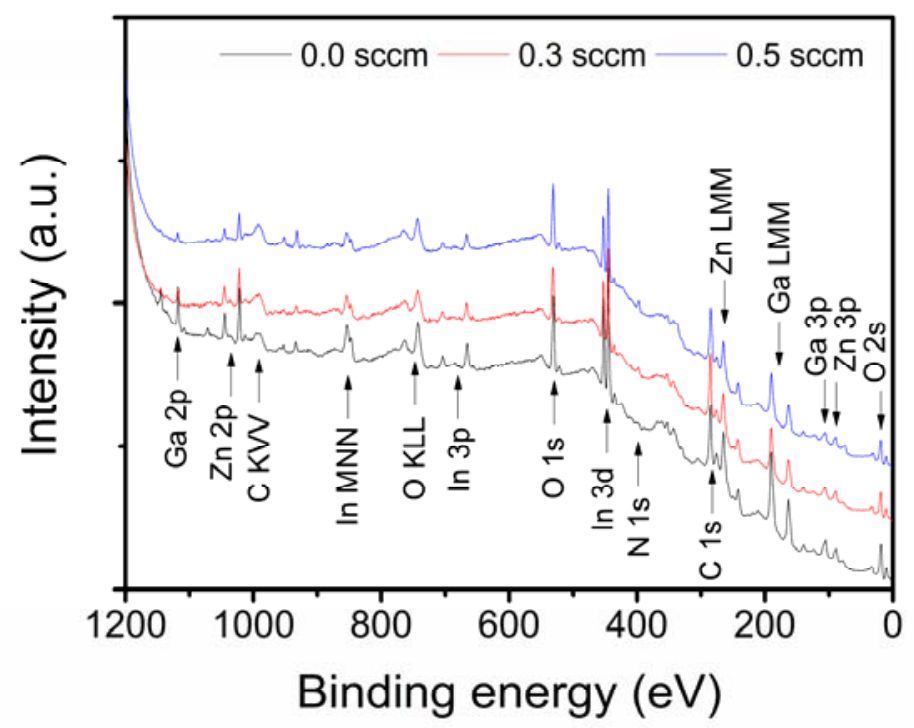

Figure S3. Wide XPS spectra of the IGZO films deposited with different $f_{\mathrm{N}_{2}}$ values. 


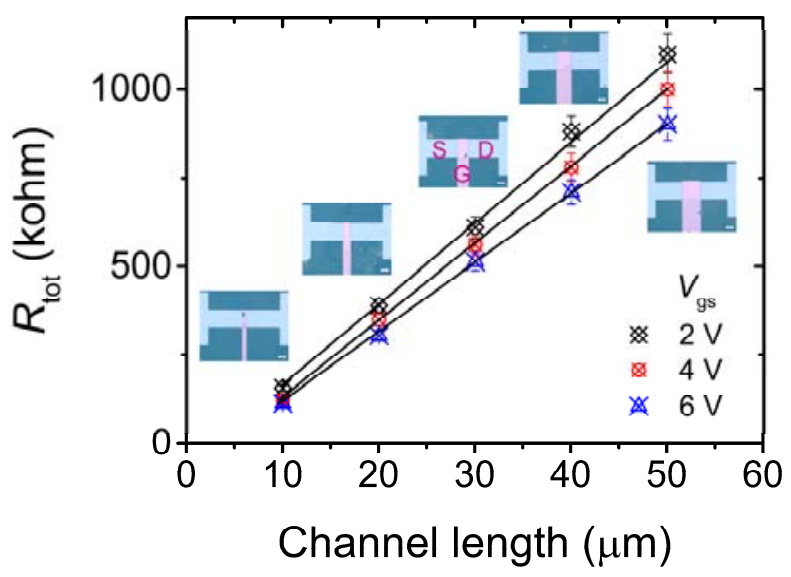

Figure S4. Channel length $(L)$-dependent resistance measured using the transmission line method for IGZO TFTs with $L$ varying from 10 to $50 \mu \mathrm{m}$. Inset top-view optical images show the actual tested devices. 


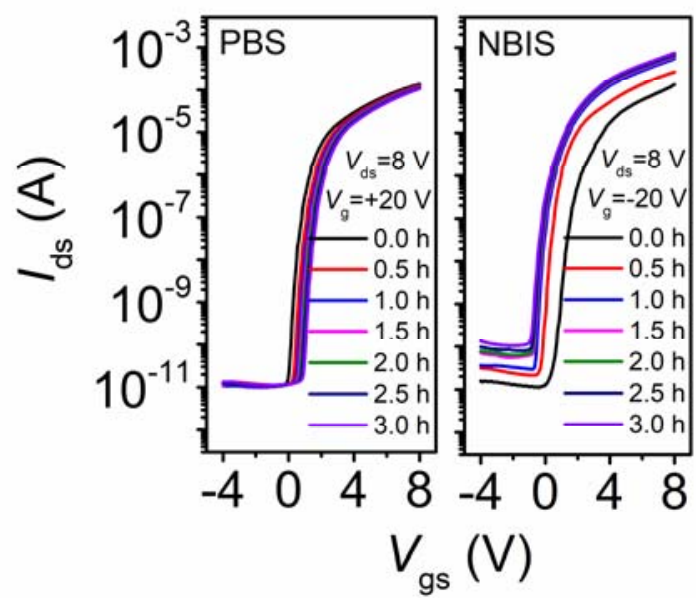

Figure S5. PBS and NBIS stabilities of the IGZO:N TFTs fabricated with $f_{\mathrm{N}_{2}}=0.8 \mathrm{sccm}$. 


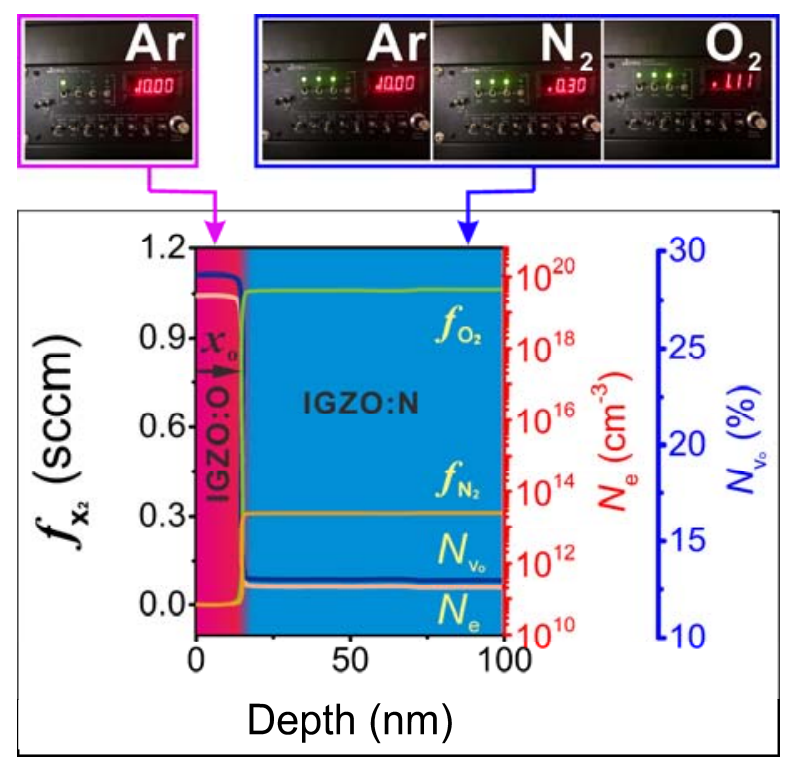

Figure S6. Variation of $f_{\mathrm{O}_{2}}$ and $f_{\mathrm{N}_{2}}$ during sequential growth of the graded-channel junctionless IGZO:O|N layer (left $\mathrm{Y}$-axis) and the corresponding spatial distributions of $V_{\mathrm{O}}$ and $N_{\mathrm{e}}$ (right $\mathrm{Y}$-Y-axes). 
(a)

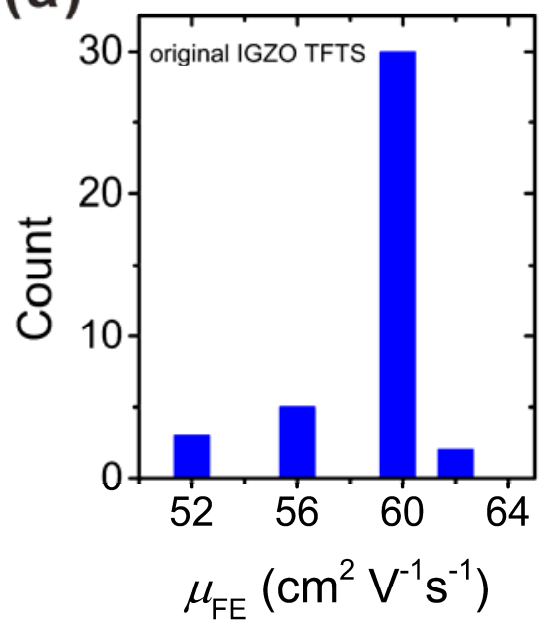

(b)

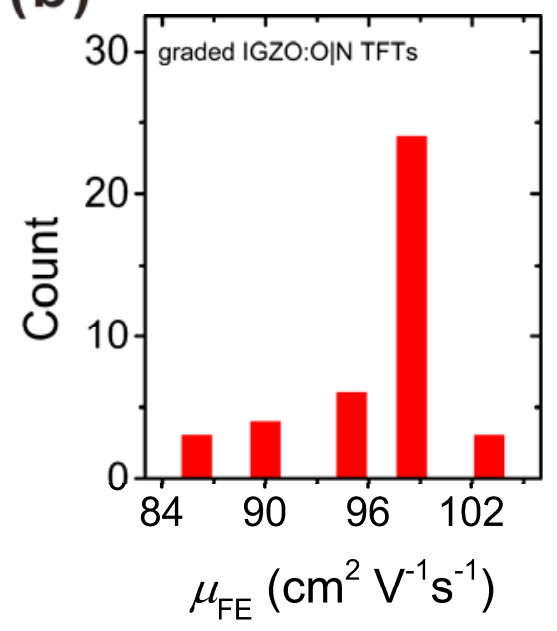

Figure S7. Statistical studies on electrical proprieties for IGZO TFTs. Field effect mobility distributions of (a) original IGZO and (b) graded IGZO:O $\mid \mathrm{N}\left(x_{\mathrm{o}}=1.5 \mathrm{~nm}\right)$ devices. 


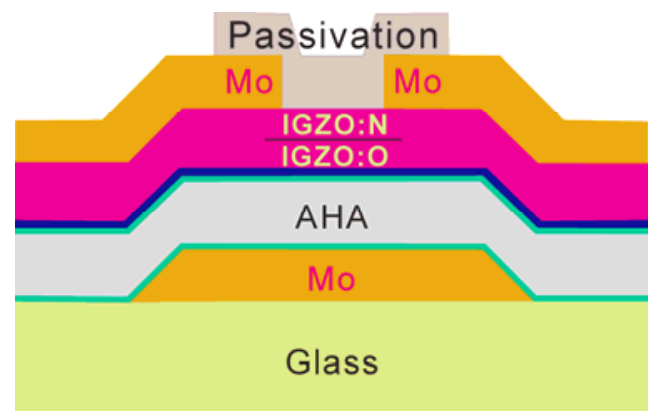

Figure S8. Schematic illustration of the device structure of bilayer-channel IGZO:O/IGZO:N TFTs. 


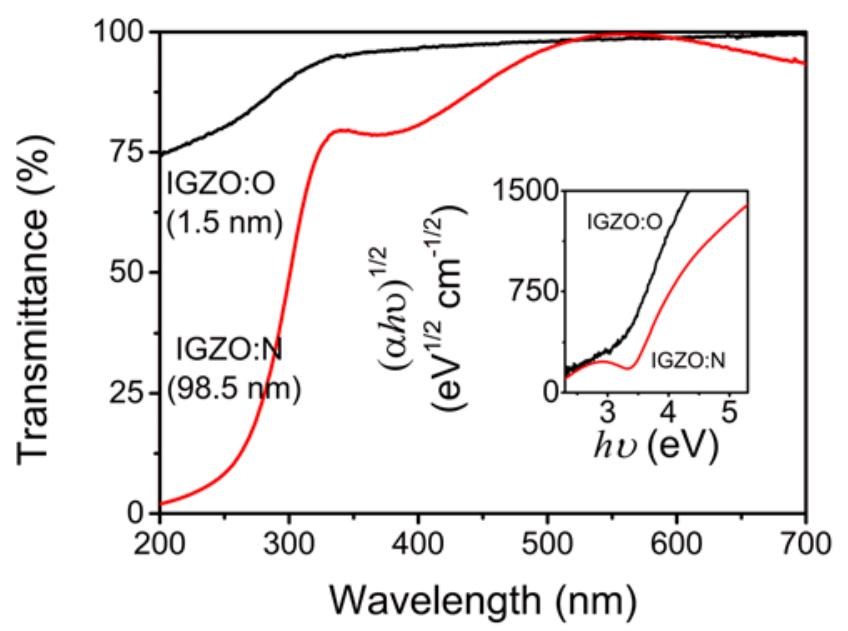

Figure S9. Optical transmittance and bandgap (inset) of the separate IGZO:O and IGZO:N channel layer grown on quartz substrates. The bandgap difference $\left(\Delta E_{\text {opt }}\right)$ between IGZO:O and IGZO:N is measured to be $0.37 \mathrm{eV}$. 


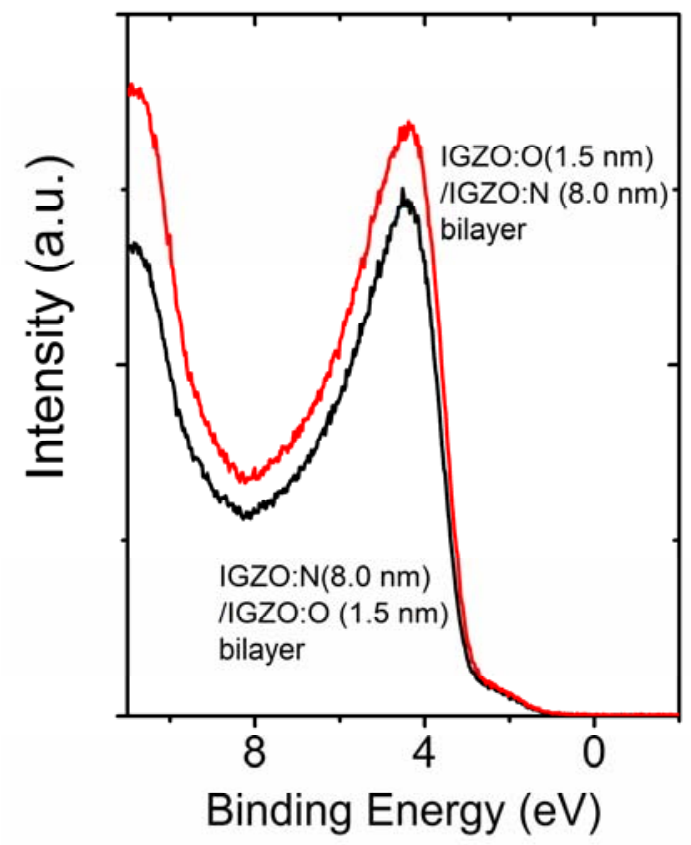

Figure S10. UPS valence band spectra of the IGZO:O $(1.5 \mathrm{~nm}) / \mathrm{IGZO}: \mathrm{N}(8.0 \mathrm{~nm})$ bilayer junction and IGZO:N (8.0 nm)/IGZO:O $(1.5 \mathrm{~nm})$ bilayer junction. To meet the sample preparation requirement for semiconductor material for UPS tests, IGZO:O $(1.5 \mathrm{~nm}) / \mathrm{IGZO}: \mathrm{N}$ $(8.0 \mathrm{~nm})$ bilayer and IGZO:N $(8.0 \mathrm{~nm}) / \mathrm{IGZO}: \mathrm{O}(1.5 \mathrm{~nm})$ bilayer were grown on conducting silver foils. After contact and charge transfer, the energy origin of the spectra is the same Fermi-level. The valence band offset $\left(\Delta E_{\mathrm{VBM}}\right)$ of $-0.10 \mathrm{eV}$ at the IGZO:O $(1.5 \mathrm{~nm}) / \mathrm{IGZO}: \mathrm{N}$ (98.5 $\mathrm{nm}$ ) junction can then be correctly calculated by the valence band energy difference of IGZO:O (1.5 nm)/IGZO:N $(8.0 \mathrm{~nm})$ bilayer and IGZO:N $(8.0 \mathrm{~nm}) / \mathrm{IGZO}: \mathrm{O}(1.5 \mathrm{~nm})$ bilayer. 